\title{
10 Incommensurability Meets Risk
}

\author{
Wlodek Rabinowicz
}

The problem I discuss in this chapter concerns interaction between incommensurability in value and risk. ${ }^{1}$ More specifically, the chapter focuses on value comparisons between risky actions whose outcomes are bound to be mutually incommensurable whatever state the world might be in. It is seemingly obvious that, in such circumstances, the actions themselves must be incommensurable in their value. Relatedly, it is seemingly obvious that one action cannot be better than another if its outcome would not be better than that of the other action, whatever state the world might be in. But these intuitions, as we shall see, can well be challenged. Indeed, they should be challenged, as there are cases in which they lead us astray.

The problem, in its main outline, was identified by Caspar Hare (cf. Hare 2010; see also Hare 2009, 2013: 45-57). It was subsequently discussed by Temkin (2012), Schoenfield (2014), and Bales et al. (2014). ${ }^{2}$ While Hare views it as a problem concerning rational preferences and rational choice between risky actions, I discuss it as a challenge for formal axiology, more specifically for a formal account of value relations. The general question is how axiology should deal with situations in which value incommensurability interacts with risk. This is also Temkin's perspective on the problem. Schoenfield and Bales et al. combine these two perspectives, i.e., the perspective of axiology and that of rational choice. They present it as a problem for rational choice guided by value comparisons. All these authors' contributions will be briefly commented on in what follows, though primarily in footnotes.

In the main text, I am going to focus on the problem itself, as I view it, and on how I think it should be solved. I will describe the problem in Section 1. In Section 2, I will present the solution I oppose, and then, in Section 3, I will present my own solution. In Section 4, I will defend my solution against a prima facie objection, and then, in Sections 5-7, I will provide an extended argument against the solution I oppose and in support of the one I favor. This argument will be based on a formal account of value relations inspired by the Fitting-Attitudes Analysis of value. In Section 8 , I will identify a limitation in the scope of my argument, a 
limitation that leads to a residual issue I address in Section 9. This issue is one I do not know how to resolve. ${ }^{3}$

\section{The Problem}

To set up the problem in its general form, consider four possible outcomes, $a, b, a^{+}$, and $b^{+}$, such that $a^{+}$is better than $a$ but not better than $b$, while $b^{+}$is better than $b$ but not better than $a$.

By the transitivity properties of betterness and equal goodness, it follows from the aforementioned that no other betterness relationships obtain between these four outcomes, nor are any of them equally good. Thus, $a$ and $a^{+}$are incommensurable both with $b$ and with $b^{+}$, where by incommensurability I mean the following value relation:

Two items are incommensurable iff neither item is better than the other nor are they equally good.

To illustrate, suppose that $a$ and $b$ are two attractive holiday options: two holiday trips to exciting, but very different, destinations. Say, $a$ is a trip to the Galápagos Islands, while $b$ is a trip to Peru; $a^{+}$and $b^{+}$are the same trips at a small discount (or simply two slightly improved trips to the same locations as $a$ and $b$, respectively). The destinations are different enough to make $a$ and $b$ incommensurable in value. The discounts (slight improvements) make $a^{+}$better than $a$ and $b^{+}$better than $b$, but they are not significant enough to make $a^{+}$better than $b$ or $b^{+}$better than $a .^{4}$

Let $S 1$ and $S 2$ be two equiprobable states of the world (two "states of nature"), exactly one of which is actually going to obtain. These states determine the outcomes of the available actions. We assume that the states per se are axiologically neutral (i.e., they are devoid of any value, either positive or negative) and, furthermore, that they do not contribute any value, positive or negative, to the outcomes they determine. Suppose, for example, that a fair coin is to be tossed and that the states are the two possible results of the toss: $S 1$ is heads and $S 2$ is tails. We assume that heads and tails both have the same objective probability of one-half.

The two states form a partition: they are mutually incompatible and jointly exhaustive. I will interpret actions in Savage's manner, as functions from states to outcomes. These are the outcomes they would cause, in different states. This way of understanding actions seems unproblematic as long as the states are causally and probabilistically independent of the actions, which I am going to assume in what follows. Indeed, I am going to assume throughout that we only consider state partitions in which the states are action-independent and are devoid of any value, whether intrinsic or contributive.

Now, consider two actions, $X$ and $Y$, and their outcomes in states $S 1$ and $S 2$, respectively: 


\begin{tabular}{lll}
\hline & $S 1$ & $S 2$ \\
\hline$X$ & $a^{+}$ & $b^{+}$ \\
$Y$ & $b$ & $a$ \\
\hline
\end{tabular}

Thus, action $X$ yields $a^{+}$in $S 1$ and $b^{+}$in $S 2$, while $Y$ yields $b$ in $S 1$ and $a$ in $S 2 .^{5}$

The problem to be discussed in this chapter is simple to pose: is $X$ better than $Y$ ? It might well seem so since both outcomes of $X$ are improvements on the outcomes of $Y .^{6}$ But it might also be argued that $X$ is not better than $Y$, since no outcome of $X$ is better than the outcome $Y$ would have in the same state. Indeed, in each state, the outcomes of $X$ and $Y$ are incommensurable. ${ }^{7}$ I will now consider the arguments on both sides in more detail: in the next section, I will focus on the view that $X$ is not better than $Y$, and in the section after that, the opposing view will be considered.

In what follows, I shall refer to the issue at hand as Hare's Problem, in honor of its originator. ${ }^{8}$ In Hare's Problem, in the form in which I pose it, incommensurability meets risk, but solving this problem does not, of course, give us anything like a complete account of the interactions between risk and incommensurability. Attempting to offer such a complete account goes far beyond the goals I have set myself in this chapter. Still, solving Hare's Problem should give us at least some opening to a more comprehensive theory.

\section{$2 X$ Is not Better than $Y$}

Assuming that states that form a given partition are causally and probabilistically independent of actions, the following principle is obviously correct:

(Statewise) Dominance: (i) An action is better than another action if, in every state, its outcome is better than that of the other action. (ii) An action is at least as good as another action if, in every state, its outcome is at least as good as that of the other action. ${ }^{9}$

Now, consider another principle that seems close in spirit to Dominance:

Complementary (Statewise) Dominance: An action is not better than another action if, in every state, its outcome is not better than that of the other action.

Or, to put it more succinctly, an action is not better than another if there is no state in which it has a better outcome. ${ }^{10,11}$

While the first clause of Dominance links better outcomes to better actions, Complementary Dominance postulates the corresponding link 
between outcomes and actions as regards the complement of betterness: the relation of not being better. If the outcomes are not better, in any state, then the action is not better.

There is also an important connection between Complementary Dominance and the second clause of Dominance: in the absence of incommensurabilities, "not better" is equivalent to "at most as good" - the converse of "at least as good." Consequently, in the absence of incommensurabilities, Complementary Dominance would collapse to the second clause of Dominance, which can also be expressed as follows: an action is at most as good as another action if, in every state, its outcome is at most as good as that of the other action. But if incommensurabilities are allowed, as in the case presented here, Complementary Dominance represents an independent constraint.

In Hare's Problem, Complementary Dominance entails that $X$ is not better than $Y$, since there is no state in which the outcome of $X$ is better than that of $Y .12$

That $X$ is not better than $Y$ would also follow if we were to accept a principle that links incommensurability of outcomes to incommensurability of actions:

Incommensurability: Two actions are incommensurable if their outcomes are incommensurable in every state.

While Incommensurability does not entail Complementary Dominance, nor is it entailed by it, the two principles are closely related in spirit. Whoever accepts one of these principles should be willing, I think, to accept the other.

It might seem that principles such as Complementary Dominance and Incommensurability are irresistible, as irresistible as Dominance. Here is how an argument for these principles might proceed.

Consider the value of an action conditional on (the obtaining of) a particular state. Arguably, this conditional value can be reduced to the value of that action's outcome in the state in question. This suggests that the same should hold for conditional value relations:

(i) The value relation between two actions that is conditional on a particular state reduces to the value relation between the outcomes of these actions in that state.

But it would seem that

(ii) If two actions stand in the same value relation conditional on each state, then this value relation must also hold between them unconditionally.

Premises (i) and (ii) imply that 
(iii) If the outcomes of two actions stand in the same value relation in each state, then this value relation must also hold between the two actions unconditionally. ${ }^{13}$

If this argument is correct, then principles such as Complementary Dominance and Incommensurability must be valid, as they are special cases of (iii), along with Dominance.

But the argument is not correct, at least not as it stands. Its conclusion (iii) is not generally true for all value relations. To see this, consider a value relation such as "not equally as good." It might well be that two actions do not stand in this relation even though their outcomes in each state do. For example, suppose you can bet on heads or on tails and gain $\$ 1$ if you win. The two bets are equally good assuming that heads are equally as probable as tails. But in every state, whether it is heads or tails, the outcomes of the bets are not equally good: one bet is won and the other is lost.

One might object that the two actions in this betting example are not equally good: one of them is better than the other, but which it is depends on the state that actually obtains. If the coin is going to fall heads up, then betting on heads is better than betting on tails, and if it is going to fall tails up, then betting on tails is better than betting on heads. This objection assumes that the value of an action depends on the actual state of the world. ${ }^{14}$ It is certainly one way of looking at an action's value, but it is not the only way. When we consider an action ex ante, before learning the state the world is in, and think of it as a potential object of choice, its outcomes in all positively probable states should play a role in determining its value - or so I would argue. However, arguing for this ex ante approach to the evaluation of actions and, more generally, for an ex ante approach to the evaluation of risky prospects would take us too far afield in this chapter. Instead, here I take the ex ante perspective for granted. The question I discuss is therefore the following: what is the right solution to Hare's Problem given the ex ante approach to evaluation? ${ }^{15}$

If (iii) is not generally true, then premise (i) or premise (ii) in the previous argument must be rejected. I am inclined to accept (i) but reject (ii). Given (i), it follows that conditionally on each state the two actions in the betting example are not equally good, because their outcomes are not equally good. But still, unconditionally, the actions are equally good, which provides a counterexample to premise (ii).

Since the previous argument, which issues in (iii), is incorrect and its conclusion is not generally true, Complementary Dominance and Incommensurability would need to be defended in some other way. As long as these principles are in doubt, we cannot conclude that, in Hare's Problem, action $X$ is not better than $Y$. 


\section{$3 X$ Is Better than $Y$}

That $X$ is better than $Y$ is the position I accept myself. I will argue for it in this section and defend this argument further in the section that follows. I also need to explain what is wrong with Complementary Dominance and will do so in Sections 6 and 7 after some necessary preparations in Section 5 .

Here, then, is how an argument for $X$ being better than $Y$ can be set up. I will first do so using very strong assumptions and then show how these assumptions can be successively weakened.

If states of nature are assigned objective probabilities, we can compare actions in terms of the lotteries on outcomes that the actions give rise to. The lottery $l_{x}$ generated by an action $x$ is a probability distribution over outcomes $o$ such that for at least one state, $S$, in the state partition, $x(S)=o$. For each such outcome $o, l_{x}(o)$ is the sum of the probabilities of the states in which $x$ yields this outcome:

$$
l_{x}(o)=\Sigma_{S} P(S)(x(S)=o),
$$

where $S$ ranges over the states in a given partition.

As is easy to see,

$$
l_{X}=\left(a^{+}, .5 ; b^{+}, .5\right) \text {, while } l_{Y}=(b, .5 ; a, .5) .
$$

In this notation, we describe a lottery by listing its possible outcomes, with each outcome being followed by its probability. The ordering in which the outcomes are listed does not matter, so, for example, $(b, .5 ; a$, $.5)$ and $(\mathrm{a}, .5 ; \mathrm{b}, .5)$ describe the same lottery.

The von Neumann-Morgenstern expected utility theory assumes that preferences for lotteries satisfy the axiom of Independence: replacing an outcome in a lottery with another outcome that is a more preferred results in a more preferred lottery. What follows is the corresponding principle for value comparisons:

Independence: For all outcomes $o, o^{\prime}, o^{\prime \prime}$ and all probabilities $p>0$, $\left(o, p ; o^{\prime \prime}, 1-p\right)$ is better than $\left(o^{\prime}, p ; o^{\prime \prime}, 1-p\right)$ iff $o$ is better than $o^{\prime}$.

According to this principle, different lottery prizes (outcomes) make independent value contributions to the value of a lottery: improving a prize always improves the lottery.

Since $a^{+}$is better than $a$ and $b^{+}$is better than $b$, Independence implies that $\left(a^{+}, .5 ; b^{+}, .5\right)$ is a better lottery than $\left(a^{+}, .5 ; b, .5\right)$ and that the latter lottery is better than $(a, .5 ; b, .5)$. Since betterness is transitive, it follows that $l_{X}=\left(a^{+}, .5 ; b^{+}, .5\right)$ is better than $l_{Y}=(a, .5 ; b, .5)$. 
Suppose we now assume:

Reduction of Actions to Lotteries: For all actions $x$ and $y, x$ is better than (equally as good as) $y$ iff $l_{x}$ is better than (equally as good as) $l_{y}$.

According to this principle, the value of an action is determined by the value of the associated lottery.

It follows from Independence and Reduction of Actions to Lotteries that action $X$ is better than action $Y$, contrary to what is implied by Complementary Dominance.

An argument for $X$ being better than $Y$ can be constructed in another way as well, without relying on such strong principles as Reduction of Actions to Lotteries and Independence. (Independence, in particular, is highly problematic. As is well-known, its preferential version has counterintuitive implications, as exemplified by the famous Allais' Problem.) Instead, we could appeal to Dominance, which we should accept in any case, together with the following weakening of Reduction of Actions to Lotteries:

Weak Reduction of Actions to Lotteries: If two actions give rise to the same lottery, they are equally good. ${ }^{16}$

Now, consider adding to $X$ and $Y$ a third action, $Z$ :

\begin{tabular}{lll}
\hline & $S 1$ & $S 2$ \\
\hline$X$ & $a^{+}$ & $b^{+}$ \\
$Y$ & $b$ & $a$ \\
$Z$ & $a$ & $b$ \\
\hline
\end{tabular}

$Y$ and $Z$ give rise to the same lottery, $(a, .5 ; b, .5)$. (Remember that the ordering of the outcomes in the description of a lottery does not matter.) Thus, $Y$ and $Z$ are equally good by the Weak Reduction of Actions to Lotteries. But by (the first clause of) Dominance, $X$ is better than $Z$ since, in every state, $X$ 's outcome is better than $Z$ 's. Since betterness is transitive across equal goodness, this means that $X$ must also be better than $Y$.

Indeed, the detour through lotteries is not needed to establish the result we are after. Instead, we can base our argument on a very simple principle that is even less demanding than Weak Reduction of Actions to Lotteries.

Switch: If for some equiprobable states $S$ and $S^{\prime}$, an action $x$ can be obtained from an action $y$ just by switching outcomes between $S$ and $S^{\prime}$, then actions $x$ and $y$ are equally good. ${ }^{17}$

In other words, a mere switch of outcomes between equiprobable states does not affect the value of an action. ${ }^{18}$ 
Now, it is easy to see that action $Z$ results from $Y$ by such a switch of outcomes between equiprobable states $S 1$ and $S 2$. Thus, Switch implies that $Y$ and $Z$ are equally good and, therefore, again by (the first clause of) Dominance and the transitivity of betterness across equal goodness, $X$ is better than $Y$, just as $X$ is better than $Z .{ }^{19} \mathrm{I}$ find this argument from Switch both simple and compelling. ${ }^{20}$

Bales et al. (2014) pose an objection to Hare's (2010) dominancebased argument for the impermissibility of $Y$, an argument in which Hare also introduces a third action $Z$ that is obtained from $Y$ by switching outcomes. ${ }^{21}$ Their objection can be adapted to my argument as well, as follows: action $Z$ would still be dominated by $X$ even if states had unequal probabilities. However, if the states' probabilities were unequal, we would no longer be able to use Switch (or Weak Reduction of Actions to Lotteries) to prove that $Y$ and $Z$ are equally good. We would thus be unable to establish that $X$ is better than $Y .{ }^{22}$ This seems unsatisfactory.

I do not find this objection very worrying. That the argument does not extend to cases in which the state probabilities are unequal is as it should be. If the difference in state probabilities were non-negligible, then it would be reasonable to judge that $X$ and $Y$ are incommensurable. Indeed, the modeling of value relations that $I$ am going to present next (beginning in Section 5) bears this out. On the other hand, it might be reasonable to expect that $X$ would still be better than $Y$ if the difference in state probabilities were very small. The argument I have presented cannot be used to prove it, but this limitation in scope does not make the argument less compelling.

I do recognize, however, that friends of Complementary Dominance will not be swayed by the argument but will reject one of its premises. Since they are committed to Dominance and also, I take it, to the transitivity of betterness across equal goodness, they will have to reject $S$ witch. Indeed, this move is perfectly natural for a friend of Complementary Dominance. As noted earlier, accepting that principle goes hand in hand with accepting Incommensurability, but Incommensurability is incompatible with Switch. Since outcomes $a$ and $b$ are incommensurable, actions $Y$ and $Z$ lead to incommensurable outcomes in every state. Therefore, Incommensurability implies that that $Y$ and $Z$ themselves are incommensurable and not equally good, as Switch would have it. In other words, from this perspective, Switch must be rejected. (The same, of course, goes for the stronger principles, such as the Reduction, or Weak Reduction, of Actions to Lotteries.) Thus, there is still work to do as long as we haven't yet provided a direct argument against Complementary Dominance and Incommensurability. I will attempt to do it in Sections 6 and 7; then I will also provide reasons for accepting Switch. 


\section{The Same Outcomes?}

First, however, we need to consider and put to rest another potential objection to the argument from Switch. The application of Switch to the case at hand assumes that the outcome of $Y$ in $S 1$ is the same as that of $Z$ in $S 2$, and - analogously - that the outcome of $Y$ in $S 2$ is the same as that of $Z$ in $S 1$. This might be questioned.

One way to question it is by arguing that an outcome includes the state in which it occurs. Thus, a more complete description of, say, the outcome of $Y$ in $S 1$ is ' $b$ in state $S 1$ ', while a more complete description of the outcome of $Z$ in $S 2$ is ' $b$ in state $S 2$ '. Conceived in this way, these two outcomes are not identical. Thus, it is not true that $Z$ is obtained from $Y$ by a mere outcome switch between equiprobable states. (For the same reason, the lotteries that $Y$ and $Z$ give rise to are not identical either.)

But this non-identity objection is ungrounded. It is perfectly reasonable to exclude from the outcomes those parts that are axiologically irrelevant. And we have assumed that the states in Hare's Problem are irrelevant in this way. While they determine the outcomes of the actions, they do not contribute any value, positive or negative, to the outcomes under consideration. Consequently, there is no reason to incorporate such states into outcomes.

A more challenging version of the non-identity objection distinguishes between seemingly identical outcomes by taking into account their modal features. In particular, if we evaluate the outcome of an action, one relevant consideration might be what the action could have brought about instead if the state of the world had been different. Think of a bet in which you lose a sum of money. For the evaluation of this outcome, it might well play a role whether the bet could have resulted in a large win. This modal fact may be relevant (positively or negatively) to the disvalue of your loss, so it may be reasonable to require that it should be included in the outcome specification.

This kind of appeal to what might have been does not, however, distinguish between the outcomes in the problem at hand. Thus, consider $b$, which is the outcome of action $Y$ in $S 1$. In the other, equally probable state, $Y$ would have resulted in $a$. The situation is exactly similar concerning $b$ as the outcome of action $Z$ in state $S 2$ : in the other, equally probable state, $Z$ would have resulted in $a$. Therefore, in this respect, there is no difference between $b$ as the outcome of $Y$ and $b$ as the outcome of $Z$. The same applies, mutatis mutandis, to outcome $a$, as brought about by $Y$ or by $Z$.

An appeal to the modal features of outcomes might, however, also take another form: in evaluating an outcome of an action, it is one thing to consider what this action might have brought about if the state had been different. But we could also consider what would or could have 


\section{0}

happened in the same state if another available action had been performed instead. It might be argued that a loss resulting from a risky action is less bad for the agent if, in a given state, it could not have been prevented by acting differently: under such circumstances, there is no reason to regret what one has done, and this might make the outcome easier to bear. ${ }^{23}$

In the problem we are considering, there is a modal difference of this kind between the otherwise identical outcomes of $Y$ and $Z$, a difference that might be relevant to their value. Thus, the outcome $b$ of $Y$ in $S 1$ is not worse than the outcome in that state of any other action at the agent's disposal. In $S 1$, actions $X$ and $Z$ would have brought about $a^{+}$and $a$, respectively, and neither of these outcomes is better than $b$. By contrast, the outcome $b$ of $Z$ in $S 2$ is worse than the outcome $b^{+}$of $X$ in the same state. Because of this modal difference, it is arguable that the outcome $b$ of $Z$ in $S 2$ is worse than the outcome $b$ of $Y$ in $S 1$. The same applies to $a$. The outcome $a$ of $Z$ in $S 1$ is arguably worse than the outcome $a$ of $Y$ in $S 2$ : in $S 1$, if the agent had chosen $X$ instead of $Z$, she would have brought about a better outcome, $a^{+}$. But in $S 2$, if the agent had chosen $X$ instead of $Y$, she would not have brought about a better outcome, as $b^{+}$is not better than $a$. This forms a basis for the objection that action $Z$ is worse than action $Y$ : each outcome of $Z$ is worse than the seemingly identical outcome of $Y$. Consequently, even though, by Dominance, $X$ is better than $Z$, it does not follow that $X$ is better than $Y$.

The objection just presented is controversial since it is debatable whether the modal features of outcomes should be seen as relevant to their value. However, there is a way to finesse the objection instead of meeting it head on: we can simply change the example by introducing a fourth action, $U$, that is related to $Z$ in the same way as $X$ is related to $Y$ :

\begin{tabular}{lll}
\hline & $S 1$ & $S 2$ \\
\hline$X$ & $a^{+}$ & $b^{+}$ \\
$Y$ & $b$ & $a$ \\
$Z$ & $a$ & $b$ \\
$U$ & $b^{+}$ & $a^{+}$ \\
\hline
\end{tabular}

By introducing $U$, we make $Y$ and $Z$ perfectly symmetrical in their modal properties: we make their corresponding outcomes modally identical. Just as for $Z$ there is another action, $X$, that in every state has a better outcome, so it is for $Y$ in our modified example: there is another action, $U$, that in every state has a better outcome. Thus, $a$ as the outcome of $Y$ and $a$ as the outcome of $Z$ now have the same modal features, as do $b$ as the outcome of $Y$ and $b$ as the outcome of $Z$.

Thus, in the presence of this fourth action, modal considerations no longer pose any obstacle to applying Switch to $Y$ and $Z$ and concluding 
that these two actions are equally good. In combination with Dominance and the transitivity of betterness across equal goodness, we can then draw the conclusion that $X$ is better than $Y$.

Someone might object at this point: your argument from Switch shows that $X$ is better than $Y$ when $Z$ is available, and you have now improved the argument by bringing in the fourth option, $U$. But how is this supposed to show that $X$ is better than $Y$ when no other alternatives are on offer, as is the case in Hare's original problem? ${ }^{24}$ I agree that the argument does not show this if one believes that the value of an action might depend on what the agent could have done instead. Note, however, that Complementary Dominance implies that $X$ is not better than $Y$, even if $Z$ and $U$ are available - that is, even in the case in which the argument from Switch cannot be faulted on modal grounds. Consequently, this argument at least shows that Complementary Dominance must be rejected if Switch is accepted.

In what follows I will simplify my discussion by assuming that the modal features of actions are not relevant to their value. On this assumption, it does not matter for the comparison of $X$ with $Y$ whether or not they are the only alternatives on offer. The argument from Switch can then be used to draw the conclusion that $X$ is better than $Y$ quite generally, independently of what other actions might be available. However, Complementary Dominance, which might seem at least as compelling as Switch, implies that this conclusion is false. Therefore, if we want to hold on to the view that $X$ is better than $Y$, we need to confront the opposition head on and take a closer look at Complementary Dominance. Is this principle as compelling as it seems to be at first sight?

\section{Value Relations Analyzed}

To approach this issue, it is helpful to ask first how value relations should be analyzed. In Rabinowicz (2008), I proposed such an analysis in the spirit of the Fitting-Attitudes account of value. According to this account, to be valuable is to be a fitting target of a pro-attitude. An item $i$ is valuable iff it is fitting (appropriate, suitable) to have a pro-attitude toward $i$. Or, to put it in more standard normative terms, $i$ is valuable iff one ought to have a pro-attitude toward $i{ }^{25}$ Different kinds of value correspond to different kinds of fitting pro-attitudes. Items that exhibit "thick" values, that are, say, admirable or delightful, call for "thick" pro-attitudes: admiration, delight. "Thin" values, such as goodness, correspond to "thin" pro-attitudes: approval or appreciation. For thin value relations, such as betterness, the relevant pro-attitude is preference: an item $i$ is better than an item $j$ iff one ought to prefer $i$ to $j$. Analogously, $i$ and $j$ are equally good iff one ought to equi-prefer $i$ and $j$, i.e., be indifferent between them. Consequently, $i$ and $j$ are incommensurable iff one neither ought to prefer one to the other nor ought to be indifferent. The kind 


\section{Wlodek Rabinowicz}

of preference and indifference that is relevant in this context is meant to be a relatively stable and considered stance. Betterness and equal goodness are thus analyzed in terms of normative requirements regarding considered preferential attitudes. These normative requirements might in some cases leave leeway for different preferences: if divergent preferences regarding $i$ and $j$ are permissible (not unfitting), then these items are incommensurable.

Let $\mathbf{I}$ be the domain of items that are being compared and suppose that $\mathbf{K}$ is the non-empty class of all permissible preference orderings of that domain. We assume that in every ordering in $\mathbf{K}$, weak preference (i.e., preference-or-indifference) is a reflexive and transitive relation. Preference and indifference are definable in terms of weak preference in the standard way, as, respectively, the asymmetric and symmetric parts of that relation. This implies, for the orderings in $\mathbf{K}$, that preference is transitive and asymmetric, while indifference is transitive, symmetric, and reflexive. We can now define different value relations on $\mathbf{I}$ in terms of $\mathbf{K}$. For any two items $i$ and $j$ in $\mathbf{I}$,

$i$ is better than $j$ iff in every ordering in $\mathbf{K}, i$ is preferred to $j$,

which is another way of saying that one ought to prefer $i$ to $j$, and

$i$ is equally as good as $j$ iff in every ordering in $\mathbf{K}, i$ and $j$ are equi-preferred,

which is another way of saying that one ought to equi-prefer $i$ and $j$.

These definitions imply that the relation of betterness-or-equal goodness is transitive and reflexive because weak preference has been assumed to be transitive and reflexive. In its turn, this entails that betterness is transitive and asymmetric, while equal goodness is transitive, symmetric, and reflexive. It also implies that betterness is transitive across equal goodness, i.e., that $i$ is better than $j$ if $i$ is better than $k$ and $k$ and $j$ are equally good. We were assuming this property of betterness in our argument from Switch in Section 3: $X$ is better than $Y$ if $X$ is better than $Z$ and $Z$ and $Y$ are equally good.

Given this modeling of value relations,

$i$ and $j$ are incommensurable iff

(i) there are orderings in $\mathbf{K}$ in which $i$ is not preferred to $j$,

(ii) there are orderings in $\mathbf{K}$ in which $j$ is not preferred to $i$, and

(iii) there are orderings in $\mathbf{K}$ in which $i$ and $j$ are not equi-preferred.

Clauses (i)-(iii) amount, respectively, to three conditions: $i$ is not better than $j, j$ is not better than $i$, and $i$ and $j$ are not equally good. 
Note that these necessary and jointly sufficient conditions of incommensurability are satisfied if (though not only if) the following holds:

$\mathrm{K}$ contains two preference orderings such that, in one, $i$ is preferred to $j$, and in the other, $j$ is preferred to $i$.

In Rabinowicz (2008), I suggested that this condition can be used to define a very common form of incommensurability that Ruth Chang calls "parity" (cf. Chang 2002). Accordingly,

$i$ and $j$ are on a par iff

it is permissible to prefer $i$ to $j$ and likewise permissible to prefer $j$ to $i^{26}$

Cases like this may arise when the comparison between two items is based on weighing several relevant dimensions, or aspects, of comparison against each other. One item might rate higher than the other on some of the dimensions and lower on others. Different assignments of weights to dimensions are often admissible in such cases, giving rise to different permissible all-things-considered preference orderings of items. $\mathbf{K}$ is meant to be the class of all such permissible all-things-considered orderings. If item $i$ comes higher than $j$ given some admissible assignments of weights to the dimensions of comparison, while $j$ comes higher than $i$ given some other admissible weight assignments, then $\mathbf{K}$ will contain preference orderings in which $i$ is ranked above $j$ and other preference orderings in which $j$ is ranked above $i$. We will thus have a case of parity.

\section{Deconstructing Complementary Dominance: The Argument from Ordinal Distances}

Consider what this analysis of value relations in terms of permissible preference orderings implies for the problem at hand. We let the item domain I consist of outcomes and actions ${ }^{27}$ and we take $\mathbf{K}$, as before, to be the class of permissible preference orderings of I. If Dominance and Switch are to be valid, then their preferential variants must hold for every ordering in $\mathbf{K}$ :

Dominance (P): (i) An action is preferred to another action if, in every state, its outcome is preferred to that of the other action. (ii) An action is weakly preferred to another action if, in every state, its outcome is weakly preferred to that of the other action. ${ }^{28}$

Switch (P): If an action $x$ can be obtained from an action $y$ just by switching outcomes between two equiprobable states, then $x$ and $y$ are equi-preferred. ${ }^{29}$ 


\section{Wlodek Rabinowicz}

Here and in what follows, "(P)" after the name of a condition stands for preference, to distinguish such conditions on permissible preferences from the analogous principles for value relations.

Now, what about Complementary Dominance? For this principle to be valid, $\mathbf{K}$ would need to satisfy the following rather clumsy condition on permissible preferences:

(*) If for every state $S$ there is some ordering in $\mathrm{K}$ in which the outcome of action $x$ in $S$ is not preferred to the outcome of action $y$ in $S$, then in some ordering in $\mathbf{K}, x$ is not preferred to $y .{ }^{30}$

Note that (*), unlike Dominance $(\mathrm{P})$ or Switch $(\mathrm{P})$, is not a constraint on each preference ordering in $\mathbf{K}$ considered separately, but instead a condition on class $\mathbf{K}$ taken as a whole: it requires that $\mathbf{K}$ contain an ordering with a certain property if $\mathbf{K}$ contains orderings exemplifying certain other properties.

While (*) is both necessary and sufficient for Complementary Dominance, it lacks immediate appeal and, indeed, it seems to lack intuitive support. To see this, consider, as an illustration, a simple model that represents the setup of Hare's Problem. There are just four outcomes in I in this model: $a^{+}$, which is better than $a$, and $b+$, which is better than $b$, but $a^{+}$is not better than $b$, nor is $b^{+}$better than $a$. Suppose that class $\mathrm{K}$ consists of three preference orderings, $P 1, P 2$, and $P 3$, that order the four outcomes as follows (in each ordering the higher-ranked outcomes appear higher up):

\begin{tabular}{lll}
\hline$P 1$ & $P 2$ & $P 3$ \\
\hline$a^{+}$ & $b^{+}$ & $a^{+}, b^{+}$ \\
$a$ & $b$ & $a, b$ \\
$b^{+}$ & $a^{+}$ & \\
$b$ & $a$ & \\
\hline
\end{tabular}

While $P 1$ and $P 2$ order the four outcomes linearly, $P 3$ contains two ties: $a^{+}$and $b^{+}$are equi-preferred in $P 3$ and so are $a$ and $b$.

Given our specification of $\mathbf{K}$, it follows that $a^{+}$is better than $a$ (as it is preferred to $a$ in every ordering in $\mathbf{K}$ ), $b^{+}$is better than $b$ (for the corresponding reason), but $a^{+}$is not better than $b$ ( $b$ is preferred to $a^{+}$in $P 2$ ), nor is $b^{+}$better than $a$ ( $a$ is preferred to $b^{+}$in $P 1$ ), just as we wanted.

P3 is not really needed for our purposes: if we removed it from $\mathrm{K}$, the model would equally well represent the assumed betterness relationships between outcomes. But adding P3 does not hurt. Indeed, instead of making $\mathbf{K}$ smaller, we could make it larger: we could add further orderings to $\mathbf{K}$, as long as in all of them $a^{+}$is preferred to $a$ and $b^{+}$is preferred to $b$. We assume for the time being, however, that all the orderings in $\mathbf{K}$ are 
complete, i.e., contain no preferential gaps. I am going to consider incomplete orderings in the next section.

The orderings in $\mathbf{K}$ extend to all the items in the domain I. Since we have assumed that $I$ also contains actions, we need to say something about how P1-P3 order actions $X, Y$, and $Z$. We need to do this in order to assess Complementary Dominance. How compelling is this principle, or - which amounts to the same thing in our analysis - how compelling is its preferential version $(*)$ ?

The answer is that it is not compelling at all. The model described earlier can serve as an illustration. The antecedent of Complementary Dominance holds for $X$ and $Y$ in this model: there is no state in which $X$ has a better outcome than $Y$. Or, what amounts to the same thing, the antecedent of $\left({ }^{*}\right)$ is satisfied by $X$ and $Y$ : for every state, there is a preference ordering in $\mathrm{K}$ in which the outcome of $X$ in that state is not preferred to the outcome of $Y$ (for state $S 1, P 2$ has this feature since it ranks $a^{+}$below $b$, while $P 1$ has this feature for $S 2$ since it ranks $b^{+}$below a). What about the consequent of $(*)$ then? Is it satisfied by $X$ and $Y$ ? Is there an ordering in $\mathrm{K}$ in which $X$ is not ranked above $Y$ ?

Note that in every preference ordering in $\mathbf{K}$, at least one of the possible outcomes of $X$, if not both, is preferred to the outcome of $Y$ in the same state. In $P 1$ this applies to $a^{+}$, the outcome of $X$ in $S 1$, in $P 2$ it applies to $b^{+}$, the outcome of $X$ in $S 2$, while in $P 3$ it applies to the outcomes of $X$ in both states. Thus, in each of these permissible orderings, the preference for $X$ over $Y$ is supported by the preference for at least one of $X$ 's possible outcomes over the corresponding outcome for $Y$.

Admittedly, in orderings $P 1$ and $P 2$ (though not in P3), the opposite preference, for $Y$ over $X$, is also supported by the preference for one of the outcomes of $Y$ over the corresponding outcome of $X$. But, as I will now argue, this support for $Y$ is weaker than the support for $X$. To see this, first consider P1:

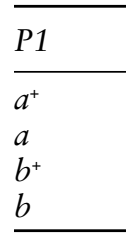

In this ordering, the preference for $X$ over $Y$ is supported by the preference for $X$ 's outcome over Y's outcome in $S 1$, i.e., in $P 1, a^{+}$is preferred to $b$. The preference for $Y$ over $X$ is supported in $P 1$ by the preference for $Y$ 's outcome over the outcome of $X$ in $S 2$, i.e., $a$ is preferred to $b^{+}$. But it is easy to see that this support for the preference for $Y$ is weaker - less pronounced - than the support for the preference for $X$. In a very intuitive sense, the ordinal distance between $a^{+}$and $b$ in $P 1$ is greater than the ordinal distance between $a$ and $b^{+}$. The former items occupy in this 


\section{Wlodek Rabinowicz}

ordering the first and the fourth place, respectively, while the latter two items occupy, respectively, the second and the third place: they are thus closer to each other.

While intuitive, this idea of being closer in an ordering can be made more precise. An assignment of numbers to items represents an ordering of the items iff higher numbers are given to items that are placed higher up in this ordering. ${ }^{31}$ Now, clearly, in every assignment that represents $P 1$, the absolute difference between the numbers assigned to $a^{+}$and $b$ must be greater than the absolute difference between the numbers assigned to $a$ and $b^{+}$, simply because of their placement in the ordering. In this sense, then, the ordinal distance between $a^{+}$and $b$ in P1 is greater than that between $a$ and $b^{+}$.

To put it more generally, for any complete ordering $P$, the ordinal distance between items $i$ and $j$ is greater than (equal to) the ordinal distance between items $k$ and $l$ iff for every assignment $v$ of numerical values to the items in P's domain, if $v$ represents $P$, then the absolute difference between $v(i)$ and $v(j)$ is greater than (equal to) the absolute difference between $v(k)$ and $v(l)$. To put it differently, when we talk about ordinal distances, we focus only on those distance comparisons that are invariant under all monotonic (order-preserving) transformations of the measurement scale.

Obviously, comparisons of ordinal distance are extremely gappy. For this reason, the notion of ordinal distance has very limited use. Thus, for example, the ordinal distance between items that occupy, say, the first and second places in an ordering is neither greater nor smaller than that between items that come in the second and fourth places. Nor are these distances equal; instead, they are incommensurable. As we have seen, however, some ordinal distances are greater or smaller than others. These differences in ordinal distances give rise to the differences in support. The support $P 1$ offers to $X$ in $S 1$ is stronger than the support it offers to $Y$ in $S 2$, because, in $P 1$, the ordinal distance between $a^{+}$and $b$ is greater than that between $a$ and $b^{+}$.

The argument with respect to $P 2$ is exactly analogous.

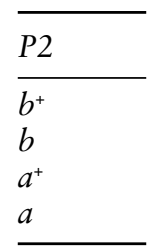

In $P 2$, just as in $P 1$, the support that the preference for $Y$ receives in one state $(S 1)$ is weaker, in terms of the ordinal distance, than the support that the preference for $X$ receives in the other state $(S 2)$. But then, since the states are equiprobable, it should not come as a surprise that $X$ is preferred to $Y$ in both $P 1$ and $P 2$, and indeed in every ordering in $\mathrm{K}$. 
(In P3, the preference for $Y$ receives no support at all; the preference for $X$ over $Y$ is supported in both states.) This means that the consequent of (") is false, or - which amounts to the same thing - that Complementary Dominance fails to hold in this model.

Indeed, we can establish a stronger conclusion. The argument from ordinal distances generalizes: in any complete preference ordering in which (i) $a^{+}$is ranked above $a$ and (ii) $b^{+}$is ranked above $b$, the support for the preference for $X$ over $Y$ is stronger, in terms of ordinal distance, than the support for the preference for $Y$ over $X .^{32}$ Unless (i) and (ii) hold, the ordering is not permissible given our assumptions about the betterness relationships between these four outcomes. Therefore, in every permissible complete ordering, $X$ is preferred to $Y$. If all preference orderings in $\mathbf{K}$ are complete, it follows that in all of them, $X$ is ranked above $Y$. Which means, on our analysis of value relations, that $X$ is better than $Y$. Thus, Complementary Dominance fails in all models of Hare's Problem in which $\mathrm{K}$ is a set of complete preference orderings. In all of them, $X$ is better than $Y$. Note, by the way, that this argument also implies that Incommensurability fails in the same way. While the outcomes of actions $X$ and $Y$ are incommensurable in every state, these actions themselves are commensurable: $X$ is better than $Y$.

A similar argument from ordinal distances can be used to defend Switch. As we know, this condition is equivalent to Switch (P). If an action is obtained from another action just by switching outcomes between equiprobable states, then, according to Switch (P), the two actions must be equi-preferred in every ordering in K. To see how we can argue for Switch $(\mathrm{P})$, let us use, as an illustration, the comparison between $Y$ and $Z$. Once again, we only consider, for the time being, complete preference orderings. In any such ordering, either (1) outcomes $a$ and $b$ are tied, and then the ordering will rank $Y$ and $Z$ equally, or (2) one of these outcomes is ranked above the other: for example, $a$ is ranked above $b$. (The opposite ranking can be dealt with in the same way.) Then (i) Y's outcome is preferred to that of $Z$ in $S 2$ and (ii) $Z$ 's outcome is preferred to that of $Y$ in $S 1$. The support that the preference for $Y$ over $Z$ gets from (i) is exactly equal to the support the preference for $Z$ over $Y$ gets from (ii); the ordinal distance from $a$ to $b$ is the same as that from $b$ to $a$. Since $S 1$ and $S 2$ are equiprobable, it follows that $Y$ and $Z$ will be equi-preferred in any such permissible ordering, just as in the permissible orderings considered in case (1). It follows, then, that Switch (P) must be satisfied by any permissible complete preference ordering. Therefore, in all models in which $\mathrm{K}$ only contains complete orderings, Switch will be valid.

Let us return to Hare's Problem. I have argued that in every permissible complete ordering, $X$ will be ranked above $Y$. This argument depends on the equi-probability of states. To see this, consider $P 1$ as an example. If $S 2$ were more probable than $S 1$, the support for the preference for $Y$ over $X$ in $P 1$, while less pronounced in terms of ordinal distance than the 


\section{8}

support for the opposite preference, would gain weight: it would come from the preference for the outcome of $Y$ in the more probable state $(S 2)$. Correspondingly, the support for $X$ over $Y$ in $P 1$, while more pronounced, would lose weight: it would come from the preference for the outcome of $X$ in the less probable state $(S 1)$. This could lead to $Y$ being ranked above $X$ in $P 1$. In $P 2$, on the other hand, the support for $X$ would gain weight and thus would even more easily outbalance the less pronounced support for $Y$. As a result, if the states had unequal probabilities, $X$ and $Y$ might well be on a par, with each of them being preferred to the other in some permissible preference ordering.

Does this mean, then, that the case in which state probabilities are equal is exceptional, a kind of singularity? That as soon as they are not equal, $X$ and $Y$ become incommensurable? This suggestion would go too far. After all, there might be cases in which all permissible preference orderings are based on cardinal representations of outcome utilities and on the associated expectational representations of the utilities of actions. In all such orderings, $X$ will still be preferred to $Y$ even after a slight perturbation of state probabilities: expected utility is continuous in both utilities and probabilities. The argument from ordinal distances presented earlier does not, of course, presuppose the expected utility interpretation of permissible preferences. The comparison of ordinal distances on which this argument relies is much less exacting than the interval measurement of preference strength needed for determining expected utilities. Still, the ordinal modeling I rely on does not exclude such interval measurement: it is in principle compatible with the expected utility interpretation of permissible preferences. Thus, even if one state becomes slightly more probable than the other, $X$ might well remain a better action than $Y$.

\section{Incomplete Preference Orderings}

There is a natural line of defense available to the friends of Complementary Dominance and Incommensurability. In the argument from ordinal distances, I have been assuming that all the preference orderings in $\mathbf{K}$ are complete. But this need not of course be the case. Indeed, in Rabinowicz (2008), I stressed that some orderings in $\mathbf{K}$ might well contain preference gaps. In Hare's Problem, a permissible preference ordering might be partly incomplete in its ranking of the four outcomes $a^{+}, a, b^{+}$, and $b$, as long as it ranks $a^{+}$above $a$ and $b^{+}$above $b$. It might contain gaps when it comes to all the other comparisons between these four outcomes. In such an incomplete ordering, none of the possible outcomes of $X$ is preferred to the outcome that $Y$ would have in the same state. Consequently, the preference for $X$ over $Y$ is not supported in this ordering by preferences for outcomes. ${ }^{33} \mathrm{~A}$ critic of my argument could therefore insist that this permissible incomplete ordering is not going to rank $X$ above $Y$ : instead, it will contain a preference gap with respect to these two 
actions. The presence of such an ordering in $\mathbf{K}$ will therefore imply that $X$ is incommensurable with $Y$. Thus, Complementary Dominance and Incommensurability will be rehabilitated.

This is certainly a weak point in my argument. But can I respond to the critic? I think so. To begin with, gaps in preference orderings might be permissible, but they do not have to be permissible. In a model in which preferential gaps among the outcomes of $X$ and $Y$ are not permissible, the argument from the preceding section shows that Complementary Dominance and Incommensurability are violated. Second, even in models with permissible preferential gaps, it might well be possible to fill in the gaps in permissible ways. To illustrate, I might, permissibly, have no preference regarding the two holiday trips, to the Galápagos Islands and to Peru, but it certainly is permissible for me to form a definite preference for one trip or the other. Likewise, it is permissible for me to form an equi-preference for these two trips. In other words, a permissible incomplete preference ordering can be permissibly completed, in various ways.

Consider where this idea can take us. Let $G$ be a set of items in the domain $I$ and $P$ a permissible preference ordering that contains gaps between some of the items in $G$. Suppose that these gaps can be permissibly filled, i.e., that there is a permissible completion of $P$ with respect to $G$, a permissible ordering $P$ ' that agrees with $P$ but is complete over $G{ }^{34}$ If such a permissible completion of $P$ with respect to $G$ exists, then it is reasonable to expect that $P$ is the common part of some set of its permissible completions with respect to $G$. In other words:

If there is a permissible completion of $P$ with respect to $G$, then $P$ is the intersection of some non-empty set of permissible completions of $P$ with respect to $G .^{35}$

If this condition is satisfied by $P$ with respect to every subset $G$ of $\mathbf{I}$, we shall say that $P$ is well-rounded. Well-roundedness is a very plausible constraint on permissible orderings. It expresses the intuition that, if the gaps in a permissible preference ordering can be permissibly filled in, then this ordering represents the preferences of someone who remains neutral when it comes to different permissible ways of filling the gaps. It contains only what is common to the different completions. ${ }^{36}$

If we accept the constraint of well-roundedness on the orderings in $\mathbf{K}$ and accept that gaps in permissible orderings can be permissibly filled in, then the argument from ordinal distances can be extended to incomplete orderings in K. If a permissible ordering $P$ is gappy within the set $\left\{a, a^{+}, b, b^{+}\right\}$, but these gaps can be permissibly filled in, then $P$ is the intersection of a set of its permissible completions with respect to this outcome set. But we already know that in all permissible orderings that are complete with respect to $\left\{a, a^{+}, b, b^{+}\right\}$, action $X$ is preferred to action $Y$. Since $P$ is the 
common part of a set of such orderings, the same must apply to $P$ itself, and even in this incomplete ordering, $X$ must be preferred to $Y$.

This means that the critic's objection fails. Complementary Dominance and Incommensurability are not satisfied even if incomplete orderings are allowed in $\mathrm{K}$.

The argument I have just presented can also be used to show that Switch ( $\mathrm{P})$ (and thus also Switch) holds even if $\mathbf{K}$ contains incomplete preference orderings. If an action $x$ can be obtained from an action $y$ by a mere switch of outcomes between equiprobable states, then it does not matter that in some permissible orderings $P$ there is a preference gap between these outcomes. As long as it is permissible to fill this gap, wellroundedness implies that $P$ will be the intersection of complete permissible orderings. We already know that $x$ and $y$ are ranked equally in all these orderings, so they will also be ranked equally in $P$.

At this point, let me digress. Complementary Dominance has an exact analogue for permissible preferences stating that for all orderings in $\mathbf{K}$, the following holds:

Complementary Dominance $(\mathbf{P})$ : An action is not preferred to another action if there is no state in which its outcome is preferred to that of the other action.

Unlike (*), this principle is neither necessary nor sufficient for Complementary Dominance..$^{37}$ But it might appear to be as compelling as the latter. Are the appearances misleading even in this case? The answer is yes. As seen earlier, in a permissible incomplete ordering according to which there is no state in which the outcome of $X$ is ranked above the outcome of $Y, X$ still must be ranked above $Y$, provided that the gaps in the ordering can be permissibly filled in and the constraint of well-roundedness is satisfied. ${ }^{38}$ However, it should be noted that Complementary Dominance $(\mathrm{P})$ is perfectly innocuous if the preference ordering under consideration is complete. Then this condition immediately follows from the second clause of Dominance (P). This is exactly analogous to the observation made in Section 2: if the value ordering is complete (i.e., if there are no incommensurabilities), then Complementary Dominance follows from the second clause of Dominance.

\section{Incomparability}

Do we now have a satisfactory general solution to Hare's Problem? Is an action such as $X$ necessarily better than its competitor $Y$ ? Well, perhaps not. The kind of case I have so far been considering is one in which potential gaps in a permissible preference ordering of outcomes can be permissibly filled in. I have considered a case in which outcomes $a^{+}$and $a$ are on a par with outcomes $b^{+}$and $b$. Even if preferential 
gaps regarding these outcomes might be permissible, it is permissible to prefer any of the former outcomes to any of the latter and vice versa. Parity is a form of incommensurability, in fact, the most common one. It is exemplified by such comparisons as the one between holiday trips, or by Hare's own comparison between different restaurant options. Arguably, it also is exemplified by another case he considers, one in which I have to choose which of my possessions should be saved from a fire: a Fabergé egg or an album with my wedding photos (cf. Hare 2010: $237 f) .{ }^{39}$

At least in principle, though, there might also exist cases of incommensurability in which it is not permissible to prefer one item to the other or to be indifferent. ${ }^{40} \mathrm{In}$ such cases what is required is a preferential gap. In Rabinowicz (2008), I refer to such a form of incommensurability as incomparability in value. Clearly, incomparability should be expected to obtain between items belonging to different ontological categories, say, between persons and events or between abstract entities such as properties or states of affairs and concrete things. But if the compared items belong to the same category, postulating incomparability is considerably more problematic. In particular, if what we compare are different action outcomes, then it is fair to say that incomparability between such items is much rarer than parity; indeed, whether it can ever occur may be disputed.

An example of Hare's Problem that might involve incomparable outcomes is provided by Bales et al. (2014). Their original example is somewhat problematic, but here is what I think is a more convincing variant of this example: ${ }^{41}$ outcomes $a$ and $b$ are, respectively, the very painful death of my mother and the very painful death of my father. In the "+"-versions of these outcomes, my mother/my father suffers slightly less when dying. $a^{+}$is slightly better (less bad) than $a$ and $b^{+}$is slightly better than $b$, but from my perspective, both $a^{+}$and $a$ are incommensurable with $b^{+}$and $b$. It might be argued that this incommensurability is of the extreme kind, that it is a case of incomparability in (personal) value. On this reading, it is impermissible for me to prefer the death of my mother (whether very painful or slightly less so) to the death of my father, and vice versa, nor is it permissible for me to be indifferent. What is required on my part is a preferential gap. Arguably, what characterizes genuine choice dilemmas is such incomparability between the alternatives. ${ }^{42}$

Are such examples as the preceding one convincing cases of incomparability of outcomes? This might be questioned. Perhaps, instead of a preferential gap being required, it might be permissible for me to be indifferent between the painful death of my mother $(a)$ and the painful death of my father $(b)$. I balk at this thought because I think that I can be indifferent only if I do not care which of them is going to die. But is this what indifference necessarily involves? It is not obvious. ${ }^{43}$ If being indifferent between two alternatives is compatible with caring 
very much which alternative is going to obtain, then the example under consideration might not be a case of incomparability: it might then be permissible for me to be indifferent rather than remain in a state of a preferential gap.

Note, however, that I should prefer my mother to die slightly less painfully if possible: the preference for $\mathrm{a}^{+}$over $b$ is one I surely ought to have. Since preferences in permissible orderings are transitive across indifference ${ }^{44}$ it would follow that I ought to prefer my mother's slightly less painful death $\left(a^{+}\right)$to the painful death of my father $(b)$ if I am indifferent between her very painful death $(a)$ and his very painful death $(b)$. This, it appears to me, is a counterintuitive implication. I ought not to have this preference in any permissible preference ordering: I ought not to prefer her death to his death (or vice versa), even if it would be slightly less painful. So, it does seem that being in a state of a preferential gap is the only permissible stance toward $a$ and $b$ in this case. If that is true, then it is a case of incomparability.

If some gaps in a preference ordering are due to incomparabilities, then such gaps cannot be permissibly filled in. But this means that in a case like this the argument from ordinal distances cannot be extended to incomplete preference orderings. This extension assumes that it is permissible to fill in preferential gaps. It is only if there is a permissible completion of a preference ordering with respect to an outcome set $G=\left\{a, a^{+}, b, b^{+}\right\}$that this ordering, by the constraint of well-roundedness, can be taken to be the intersection of a set of its permissible completions with respect to $G$.

\section{Residual Paradox: Incomparability Meets Risk}

So, where does this leave us? I have provided an argument that explains why Complementary Dominance must be rejected: one action might well be better than another even if there is no state in which it has a better outcome. I have shown how this can be possible when the outcomes of two actions are on a par in every state. But my argument does not apply to a weakening of Complementary Dominance, according to which one action cannot be better than another if there is no state in which its outcome is better than, or even comparable with, the outcome of the other action. Indeed, the following principle seems compelling:

Incomparability: Two actions are incomparable if their outcomes are incomparable in every state.

This means, however, that we still confront a paradox: Incomparability is intuitively compelling, but so is Switch, and these two principles are mutually incompatible if incomparabilities between outcomes are possible. If they are possible and Incomparability holds, then Switch fails to 
hold. Actions $Y$ and $Z$ exemplify this point: if $a$ and $b$ are incomparable, then the outcomes of $Y$ and $Z$ are incomparable in every state. But then Incomparability implies that $Y$ and $Z$ themselves are incomparable. They are not equally good, as Switch would have it.

However, Switch is no less compelling when the outcomes are incomparable. Even then it seems intuitive that switching outcomes between equiprobable states should not affect the value of an action. Why should it matter whether I obtain outcome $a$ if the coin falls heads up and outcome $b$ if it falls tails up, or vice versa? Or does it matter if $a$ and $b$ are incomparable? Even though, as I hope to have shown, it would not matter otherwise.

These questions remain with us. But then, despite the progress we have made when it comes to interactions between parity and risk, we still are left with the question concerning the interaction between risk and incomparability. We seem to have on our hands something that looks like a genuine paradox: both Incomparability and Switch are intrinsically compelling, none of them seems vulnerable to a direct criticism, but if incomparable outcomes are possible, these two principles cannot both be correct.

It is a paradox I do not know how to resolve.

\section{Notes}

1 This chapter has been long in the making: its first version was circulated as early as 2016, and it was essentially completed by 2017 . Over the years, I presented it at philosophy seminars at York University, London School of Economics, Lund University, the Research School of Social Sciences at the Australian National University in Canberra, Humboldt University in Berlin, and at a workshop on formal theories of value at the University of Colorado at Boulder. I am indebted to the participants in these events and to several other colleagues for very helpful comments and suggestions. Special thanks are due to Matt Adler, Staffan Angere, Adam Bales, Luc Bovens, Richard Bradley, John Broome, Krister Bykvist, Daniel Cohen, Franz Dietrich, Johan Gustafsson, Alan Hájek, Christian List, David Makinson, Jake Nebel, Graham Oddie, Christian Piller, Toni Rønnow-Rasmussen, Mozaffar Qizilbash, Thomas Schmidt, Katie Steele, Orri Stefánsson, and John Weymark. I also received very helpful comments from the editors of this volume, Henrik Andersson and Anders Herlitz. Finally, I want to thank Stephen Sanborn at Proper English AB for a very thorough and competent language review.

2 Three more recent articles should also be mentioned, by Bader (2018) and Doody (2019a, 2019b). They are all well worth studying but will not be commented on here, as they appeared after this chapter had been essentially completed.

3 I have been waiting to get this chapter off my chest, mainly because I continued to hope, in vain as it happens, that I would find a way to solve this residual problem. Now, however, it is time to present what I have and leave the outstanding issues to the reader.

$4 \mathrm{It}$ is not essential for the problem at hand that it is the same kind of improvement in both cases or that the size of the improvement is exactly the same (if 
it is meaningful to compare these sizes). If $a^{+}$is a trip to the Galápagos Islands at a discount, then $b^{+}$could be a trip to Peru with an extra day in Cuzco.

5 In his comments on this chapter, Anders Herlitz has suggested how this choice problem could arise in real life. Suppose $X$ and $Y$ are two vacation packages one can buy: $X$, departing on a Tuesday, is slightly cheaper than $Y$, departing on a Wednesday. Whether you fly on a Tuesday or on a Wednesday does not matter to you, but the price difference does matter, though only marginally since it is quite small. Both packages promise to take you to either the Galápagos Islands or Peru, but the destinations you get to by choosing $X$ and $Y$, respectively, are entirely dependent on whether the airline decides to fly to Quito on Tuesdays and to Lima on Wednesdays (S1) or the other way round $(S 2)$. The airline has not yet made its decision, and it may go either way, with equal probability. Vacation package $X$ involves departure on a Tuesday, which means it will take you to the Galápagos Islands via Quito $\left(a^{+}\right)$if the airline decides to fly to Quito on Tuesdays and to Lima on Wednesdays, but it will take you to Peru $\left(b^{+}\right)$if the airline makes the opposite decision. Vacation package $Y$ involves departure on a Wednesday, which means it will take you to Peru $(b)$ if the airline decides to fly to Quito on Tuesdays and to Lima on Wednesdays, but it will take you to the Galápagos Islands via Quito $(a)$ if the airline makes the opposite decision.

6 In terms of the illustration used (see the preceding endnote), why not opt for the cheaper trip if it does not matter whether you fly on a Tuesday or a Wednesday?

7 Thus, Bales et al. (2014) and Schoenfield (2014) all argue that $X$ and $Y$ are equally permissible in a case like this. Hare (2010), on the other hand, argues that it would be irrational to opt for $Y$; rationality requires that you choose $X$.

8 However, as mentioned previously, for Hare it was a problem of rational choice and not an issue in axiology. I will also sometimes use the label "Hare's Problem" for the choice situation itself in which the agent confronts actions $X$ and $Y$ specified as above $-I$ hope that this will not create any confusions. Bader (2018) rather aptly calls such choice situations cases of "opaque sweetening."

9 I have three comments: (i) this principle, like several others that follow, refers to two distinct value relations: one between outcomes and the other between actions. The former relations can be reduced to the latter if we associate each outcome with the "constant" act that assigns this outcome to every state in the partition. Then the betterness relation between outcomes is reduced to the betterness relation between associated constant acts. (ii) As a general principle, Dominance is plausible only if the states in the partition considered are independent of actions (causally or probabilistically independent, depending on the kind of decision theory we accept). In what follows, this act-independence of states will be assumed throughout. (iii) Even with actindependence, Dominance can fail if the number of states is infinite and the outcomes themselves involve elements of risk or uncertainty. A case in point is the well-known Two-Envelopes Problem (cf. Broome 1995; Dietrich and List 2005). Here we only consider cases in which the space of possibilities is partitioned into a finite number of alternative states.

10 Bales et al. (2014: 460) defend a principle of rational choice that is closely related to Complementary Dominance but applies not merely to binary choices. It is framed in terms of "not worse" instead of "not better," but this difference does not matter:

Competitiveness: An action is rationally permissible if there is no state in which its outcome is worse than that of some alternative action. 
Assuming that an action is rationally permissible iff it is not worse than any alternative action, Competitiveness follows from Complementary Dominance, though not vice versa. But in binary choices, such as Hare's Problem, the two principles are equivalent.

11 It is easy to see that Complementary Dominance entails the following principle:

Neither Nor: An action is neither better nor worse than another action if there is no state in which its outcome is better or worse than that of the other action.

In what follows, I am going to argue against Complementary Dominance, but my argument will also apply to Neither Nor.

Schoenfield (2014: 267) adopts a principle of rational choice that corresponds to the conjunction of the first clause of Dominance and Neither Nor:

Link: In cases in which considerations of value are the only ones that are relevant, if you are rationally certain that one option, $A$, will bring about greater value than the alternative option, $B$, you're required to choose $A$. If you are rationally certain that neither of the two options will bring about greater value than the other, it's not required that you choose $A$, and it's not required that you choose $B$.

12 Hare (2010), who does not discuss value relations but instead focuses on rational preferences and rational choice, considers, but in the end rejects, a principle of deference to one's "better-informed self" (p. 242). In a comparison between two actions, such as $X$ and $Y$, my better-informed self, who knows which state obtains, would have no preference in favor of either of these actions. His preferences for actions would be determined by their actual outcomes, and I know that he would not prefer the outcome of one action to that of the other, whichever state actually obtains. My better-informed self would have the same preferences regarding final outcomes as I have, and Hare assumes that I do not prefer $a^{+}$to $b$ or $b$ to $a^{+}$and, similarly, that I do not prefer $b^{+}$to $a$ or $a$ to $b^{+}$. But then, if I know that my better-informed self would have no preferences in favor of $X$ or $Y$, the principle of deference implies that it is (at least) rationally permissible for me to have no such preferences either. Clearly, this conclusion should be welcomed by friends of Complementary Dominance. (It might be noted that the preferential version of Dominance could be defended along the same deferentialist lines.)

13 Temkin (2012: section 8.3, p.139) considers and defends (with some important qualifications, see endnote 19) The State-by-State Comparison Principle:

For any two prospects [or actions] $A$ and $B$, if the value of $A$ 's outcome stands in a particular comparative relation, $R$, to the value of $B$ 's outcome for each possible state of nature, then prospect $A$ stands in relation $R$ to $B$.

This principle corresponds to (iii), and Temkin's defense of the principle relies on an argument similar to the one I outlined earlier.

14 If this state is not yet determined (before the toss of the coin), then, according to this view, it is not yet determined which of the bets is better, although it is determined that they are not equally good.

15 One should bear in mind, though, that some of the defenders of the view that, in Hare's Problem, $X$ is not better than $Y$ might take this view precisely because they adhere to the ex post approach to the evaluation of actions. 
16 This principle is identical to what Temkin (2012: section 8.3:238) calls The First Principle of Equivalence:

For any two prospects [or actions] $A$ and $B$, if for every possible outcome $[\ldots]$ that might arise with a given probability [...] if $A$ is chosen, the same outcome [...] might arise with the same probability [...] if $B$ is chosen, and vice versa, then prospects $A$ and $B$ are equally good [...].

17 Remember that the states under consideration are assumed to be axiologically irrelevant, i.e., they do not contribute any value, positive or negative, to outcomes. Without this assumption, Switch would not be a plausible principle.

18 To put it more formally, actions $x$ and $y$ are equally good if for some equiprobable states $S$ and $S^{\prime}, x(S)=y\left(S^{\prime}\right), x\left(S^{\prime}\right)=y(S)$, and for all other states $S^{\prime \prime}$ in the state partition, $x\left(S^{\prime \prime}\right)=y\left(S^{\prime \prime}\right)$. While Switch is a very simple condition with a highly limited scope of application, it is equivalent to a principle whose application is wider:

Permutation: Let $\pi$ be a probability-preserving permutation on states in a partition. If for every state $S$, the outcome that action $x$ assigns to $S$ is assigned by action $y$ to $\pi(S)$, then $x$ and $y$ are equally good.

Switch is a special case of this more general principle; it is a case in which $\pi$ is a simple transposition of one state into another that is equally probable. At the same time, this special case implies the general principle since any probability-preserving permutation on a finite set of states is the relative product of a finite sequence of probability-preserving transpositions. Consequently, by the transitivity of equal goodness, Switch entails Permutation.

19 An essentially similar argument has been presented by Temkin (2012: section 8.3). The example Temkin considers differs somewhat from the one offered by Hare. In Temkin's example, $Z$ results from $Y$ by an outcome switch, but action $X$ leads to an improved outcome as compared with $Z$ only in one state and to the same outcome in the other state. Consequently, in his argument that $X$ is better than $Z$, Temkin needs to rely on a stronger principle of dominance than the one I have been assuming. Temkin concludes from this argument that the First Principle of Equivalence (which implies that $Y$ and $Z$ are equally good) and the State-by-State Comparison Principle (which, as we have seen, implies that $X$ is not better than $Y$ ) are incompatible with each other. He finds the former principle particularly compelling. He therefore suggests that the State-by-State Comparison Principle should be restricted to accommodate the First Principle of Equivalence: "Perhaps the most natural way to do that would be to limit the scope of the State-by-State Comparison Principle to all and only those cases where it would not directly conflict with the First Principle of Equivalence" (Temkin 2012: 242). Temkin admits, however, that in the absence of justification, it is an ad hoc solution.

20 An even shorter, more direct argument for the same conclusion appeals to Stochastic Dominance as its only premise (see Bader 2018).

Stochastic Dominance: An action $x$ stochastically dominates an action $y$ iff (i) for every possible outcome $o$, the probability that $x$ will result in an outcome at least as good as $o$ is at least as great as the corresponding probability for $y$, and (ii) for some $o$, this probability is greater. If clause (i) is satisfied, $x$ is at least as good as $y$. If (i) and (ii) are satisfied, $x$ is better than $y$.

It is easy to see that $X$ stochastically dominates $Y$ and thus is better than $Y$ according to this condition. But it may be questioned whether Stochastic 
Dominance is as intuitively compelling as the principles I have used in my argument from Switch. Stochastic Dominance is in any case much stronger than Statewise Dominance and Switch taken together. Admittedly, appealing to it in the argument for $X$ being better than $Y$ obviates the need to assume that betterness is transitive across equal goodness. Although this may be seen as an advantage by some, the advantage is minimal if one finds, as I do, this transitivity condition extremely intuitive.

In private communication, Orri Stefánsson has suggested another principle that would immediately establish that $X$ is better than $Y$ :

An action $x$ is better than an action $y$ if the outcome of $x$ would in every state be better than that of $y$ if $y$ 's outcomes were switched between some equiprobable states.

This condition is much weaker than Stochastic Dominance and yet it also makes it unnecessary to appeal to the transitivity of betterness across equal goodness. But there is a price to pay: the condition is ad hoc, being especially designed to immediately get us the conclusion we are after. Jumping to the conclusion in this way is unattractive; it is preferable to proceed to it by separate steps that can be subjected to independent scrutiny.

21 Hare (2010) defines the notion of prospects associated with an action: each such prospect is a possible outcome of that action paired with the agent's credence that the action would yield the outcome in question. The set of all prospects associated with an action is thus just like a lottery but with objective probabilities replaced by credences. Hare then assumes the following principle:

Prospects Determine Permissibility: Facts about what it is rationally permissible for me to do are determined by facts about the prospects associated with the options available to me.

(Hare 2010: 240)

This principle is similar in spirit to the Reduction of Actions to Lotteries.

Hare then considers two choice situations, one in which we choose between $X$ and $Y$ (with equal objective probabilities of states replaced by equal credences) and the other in which the choice is between $X$ and $Z$. He implicitly assumes the preferential version of Statewise Dominance as a criterion of permissibility, and thus concludes that $Z$ is impermissible in the latter choice problem. This follows even if the agent has incomplete preferences, as long as she prefers $a^{+}$to $a$ and $b^{+}$to $b$. But since the prospects associated with $Y$ and $Z$ are the same and since in both choice problems the alternative action is the same $(X)$, if Prospects Determine Permissibility, $Y$ must be impermissible in the choice between $X$ and $Y$, just as $Z$ is impermissible in the choice between $X$ and $Z$.

Hare also puts forward a general theory of rational choice, Prospectism, which as a special case implies that $Y$ is impermissible if $X$ is available. That theory, however, rests on strong assumptions, much stronger than those I am willing to rely on in this chapter. According to Prospectism, an action, interpreted as a set of prospects, is permissible iff it maximizes expected utility (as compared with other available actions) with respect to some utility function on outcomes that is compatible with the agent's possibly incomplete preferences over outcomes. Needless to say, the view that an action is permissible only if it is in this way rationalizable in terms of expected utility maximization is contentious. At the same time, the theory might be criticized for being too permissive in that it imposes no restrictions on admissible utility 
functions apart from the requirement that they must be compatible with the agent's outcome preferences. In the case we consider, if the agent's preferences have gaps when it comes to comparing $a$ and $a^{+}$with $b$ and $b^{+}$, Prospectism implies that action $Y$ becomes permissible as soon as the agent ever so slightly increases her credence for one of the states. For this objection to Prospectism, see Nissan-Rozen (2015).

22 Nor would it be possible to use Stochastic Dominance to establish this conclusion. It would no longer be the case that $X$ stochastically dominates $Y$. To see this, suppose that $S 1$ is more probable than $S 2$. Then the probability that $X$ results in an outcome at least as good as $b$ is lower than the corresponding probability for $Y$. Analogously, if $S 2$ is more probable than $S 1$, the probability that $X$ results in an outcome at least as good as $a$ is lower than the corresponding probability for $Y$.

23 For a discussion of different kinds of regret and ways of allaying them, and in particular for the distinction between outcome regret, which arises when we reflect on what our action could instead have brought about, and action regret, which comes from reflecting on what would have been brought about if another action had been chosen, see Bovens and Rabinowicz (2015).

$24 \mathrm{I}$ am indebted to Anders Herlitz for pressing this point.

25 This formulation ignores various qualifications and provisos we might want to impose, for example, that an agent ought to hold a pro-attitude toward $i$ only if she knows what $i$ is like, that the "ought" in question is an ought of recommendation rather than a strict demand, or that the reasons for holding a pro-attitude (i.e., the considerations that explain why one ought to hold it) should be of the "right kind" - they should invoke value-making features of the item under consideration rather than extraneous factors (for this last point, see Rabinowicz and Rønnow-Rasmussen 2004). In what follows, I make a tacit assumption that these qualifications are satisfied.

26 This definition does not exclude, of course, that in cases of parity it might also be permissible to be indifferent between the items in question or even to have no preferential attitude as far as they are concerned. Indeed, in typical cases of parity, all these possibilities can be expected to be permissible (cf. Rabinowicz 2008).

27 As previously, we think of an action as a function from states in a state partition to outcomes. In some cases, particularly in the cases we focus on, the elements of the state partition are assigned objective probabilities. In what follows, whenever two actions are being compared, they are assumed to be based on the same state partition.

28 That the first clause of Dominance $(\mathrm{P})$ holds for all orderings in $\mathbf{K}$ is both necessary and sufficient for the corresponding first clause of Dominance. However, that the second clause of Dominance $(\mathrm{P})$ holds for all orderings in $\mathbf{K}$ is not sufficient for the second clause of Dominance. Using the former principle, we can prove that if the outcomes of $x$ are at least as good as the outcomes of $y$ in every state, then $x$ is weakly preferred to $y$ in every ordering in K. But this falls short of $x$ being at least as good as $\mathrm{y}$. To be at least as good as $y, x$ must be better than $y$ or equally as good as $y$, i.e., it must be preferred to $y$ in every $\mathbf{K}$-ordering or equi-preferred with $y$ in every $\mathbf{K}$-ordering. It is not enough if it is preferred in some orderings and equi-preferred in others. This problem would be avoided if one redefined the notion of being at least as good: $i$ could be said to be at least as good as $j$ iff $i$ is weakly preferred to $j$ in every ordering in K (cf. Rabinowicz 2008). While "at least as good" on this redefinition would still be entailed by "better or equally good," the converse entailment would no longer hold.

29 That this condition holds for all orderings in $\mathbf{K}$ is both necessary and sufficient for Switch. 
30 Or, by contraposition: if action $x$ is preferred to action $y$ in every ordering in $\mathrm{K}$, then there is some state $S$ such that the outcome of $x$ in $S$ is preferred to the outcome of $y$ in $S$ in every ordering in $\mathbf{K}$.

31 More generally, for any complete ordering $P$ of the items in a certain domain, an assignment $v$ of numerical values to the items in this domain represents $P$ iff $v$ assigns higher numbers to items placed higher in $P$ and the same numbers to items that are equal ranked in $P$.

32 In fact, $P 1$ and $P 2$ are the only permissible complete orderings in which the preference for $Y$ over $X$ receives any support at all. Proof: the preference for $Y$ over $X$ receives some support only if (1) $a$ is preferred to $b^{+}$or (2) $b$ is preferred to $a^{+}$. Since $a^{+}$is preferred to $a$ and $b^{+}$is preferred to $b,(1)$ implies, by the transitivity of preference, that the four items are ordered as in $P 1$, while (2) implies, again by transitivity, that they are ordered as in $P 2$.

At the same time, in any complete ordering, the preference for $X$ over $Y$ must receive some support. Proof: if $a^{+}$is ranked above $a$ and $b^{+}$is ranked above $b$, then, by completeness, (i) $a^{+}$is preferred to $b$ or (ii) $b^{+}$is preferred to $a$. If (i) holds, the preference for $X$ over $Y$ receives support in $S 1$. If (ii) holds, this preference receives support in $S 2$.

33 But could the preference for $Y$ over $X$ be supported in a permissible incomplete ordering without the preference for $X$ over $Y$ being supported? No, it could not. If the preference for $Y$ over $X$ is supported, this must mean that the ordering ranks (i) $a$ above $b^{+}$or (ii) $b$ above $a^{+}$. Being permissible, it ranks (iii) $a^{+}$above $a$ and (iv) $b^{+}$above $b$. By the transitivity of permissible preferences, (i), (iii), and (iv) imply that the ordering in question is identical to $P 1$, while (ii), (iii), and (iv) imply that it is identical to $P 2$. And we already know that in both these complete orderings, the preference for $X$ over $Y$ is supported and, indeed, that it is more pronounced than the preference for $Y$ over $X$.

$34 P^{\prime}$ agrees with $P$ iff for all items $i$ and $j$ in $\mathrm{I}$, if $i$ is preferred to (equi-preferred with) $j$ in $P$, then $i$ is also preferred to (equi-preferred with) $j$ in $P^{\prime}$. Note that agreement is not a symmetric relation: if $P^{\prime}$ agrees with $P, P$ might not agree with $P^{\prime}$. Indeed, this will always be the case if $P^{\prime}$ fills some gaps in $P$.

35 This condition can also be equivalently stated as follows:

If the set $\Delta(P, G)$ of all permissible completions of $P$ with respect to $G$ is not empty, then $P=\cap \Delta(P, G)$.

To establish this equivalence, it is sufficient to note that if $P$ is the intersection of a non-empty set of orderings, then adding to that set further orderings that agree with $P$ will not make any difference to the intersection.

36 A complete ordering is of course trivially well-rounded since it does not contain any gaps.

37 It is not sufficient since the antecedent of $(*)$ does not require that the antecedent of Complementary Dominance $(\mathrm{P})$ should be satisfied by any ordering in $\mathrm{K}$. Thus, Complementary Dominance $(\mathrm{P})$ cannot be used to prove the consequent of (*) from its antecedent. Nor is Complementary Dominance $(\mathrm{P})$ necessary for $(*)$ since the consequent of $(*)$ is relatively weak: it only stipulates that there exists some ordering in $\mathrm{K}$ in which action $x$ is not preferred to action $y$. We could have a model in which some such ordering exists, even though $\mathrm{K}$ also contains another ordering that violates Complementary Dominance $(\mathrm{P})$ with regard to actions $x$ and $y$.

38 This counterexample also applies to what might be seen as a kind of preferential analogue of Incommensurability, i.e., the principle that requires a preference gap between two actions if there are preference gaps between their outcomes in every state. It should be kept in mind, though, that 
incommensurabilities in value and preference gaps are not the same thing. If a preference gap between two items is permissible, this entails that the items in question are incommensurable, but the opposite implication need not hold.

39 Note, however, that Hare, who is interested in the preferential version of his problem, assumes in his examples that the agent lacks preferences regarding these options. My interest is in the value relations, so I allow both the absence of preference and its presence, although in the latter case, I assume that it is permissible to have a preference either way.

40 Or this impermissibility might at least apply to considered preferential attitudes Both considered preference and considered equi-preference might be disallowed.

$41 \mathrm{I}$ am indebted to Luc Bovens for suggesting this variant.

42 In Rabinowicz (2008), I suggested that Sophie's Choice might be an example of a situation in which the alternative outcomes are incomparable from Sophie's perspective: she must choose which of her children to save, but it is impermissible for her to prefer one child rather than the other to survive, nor is it permissible for her to be indifferent between these outcomes.

$43 \mathrm{I}$ am indebted to Graham Oddie for pressing this point.

44 That preferences in permissible orderings are transitive across indifference is a crucial assumption in my model. Without it, the model would not validate the condition that betterness is transitive across equal goodness.

\section{References}

Bader, R. M. (2018), 'Stochastic Dominance and Opaque Sweetening', Australasian Journal of Philosophy 96/3: 498-507.

Bales, A., Cohen, Daniel, and Handfield, T. (2014), 'Decision Theory for Agents with Incomplete Preferences', Australasian Journal of Philosophy 92/3: 453-470.

Broome, J. (1995), 'Two Envelope Paradox', Analysis 55/1: 6-11.

Bovens, L., and Rabinowicz, W. (2015), 'The Meaning of 'Darn It!', in I. Hirose and A. Reisner (eds.), Weighing and Reasoning: Themes from the Philosophy of John Broome, Oxford University Press, Oxford: 129-139.

Chang, R. (2002), 'The Possibility of Parity', Ethics 112/4: 659-688.

Dietrich, F., and List, C. (2005), 'The Two-Envelope Paradox: An Axiomatic Approach', Mind 114/454: 239-248.

Doody, R. (2019a), 'Opaque Sweetening and Transitivity', Australasian Journal of Philosophy 97/3: 559-571.

Doody, R. (2019b), 'Parity, Prospects, and Predominance', Philosophical Studies 176/4: 1077-1095.

Hare, C. (2009), 'Perfectly Balanced Interests', Philosophical Perspectives 23/1: 165-176.

Hare, C. (2010), 'Take the Sugar', Analysis 70/2: 237-247.

Hare, C. (2013), The Limits of Kindness (Oxford University Press).

Nissan-Rozen, I. (2015), 'Review of The Limits of Kindness by Caspar Hare', Economics and Philosophy 31/3: 493-499.

Rabinowicz, W. (2008), 'Value Relations', Theoria 74/1: 18-49.

Rabinowicz, W., and Rønnow-Rasmussen, T. (2004), 'The Strike of the Demon: On Fitting Pro-attitudes and Value', Ethics 114/3: 391-423.

Schoenfield, M. (2014), 'Decision Making in the Face of Parity', Philosophical Perspectives 28/1: 263-277.

Temkin, L. S. (2012), Rethinking the Good - Moral Ideals and the Nature of Practical Reasoning (Oxford University Press). 